\title{
The Euler Equations on Thin Domains
}

\author{
Jerrold E. Marsden \\ Control and Dynamical Systems, California Institute of Technology 107-81 \\ Pasadena, CA 91125, USA \\ Tudor S. Ratiu \\ Département de Mathématiques, Ecole Polytechnique Fédérale de Lausanne \\ CH-1015 Lausanne, Switzerland \\ Geneviève Raugel \\ Université de Paris-Sud et CNRS, UMR 8628, Mathématiques, Bât. 425 \\ F-91405 Orsay Cedex, France \\ International Conference on Differential Equations, Berlin, 1999 \\ Edited by B. Fiedler, K. Gröger and J. Sprekels, \\ World Scientific, 2000, 1198-1203.
}

\begin{abstract}
For the Euler equations in a thin domain $Q_{\varepsilon}=\Omega \times(0, \varepsilon), \Omega$ a rectangle in $\mathbb{R}^{2}$, with initial data in $\left(W^{2, q}\left(Q_{\varepsilon}\right)\right)^{3}, q>3$, bounded uniformly in $\varepsilon$, the classical solution is shown to exist on a time interval $(0, T(\varepsilon))$, where $T(\epsilon) \rightarrow+\infty$ as $\epsilon \rightarrow 0$. We compare this solution with that of a system of limiting equations on $\Omega$.
\end{abstract}

\section{Introduction}

The Euler equations in three-dimensional thin domains arise in geophysical problems such as atmospheric and ocean dynamics. For instance, in ocean dynamics, one has large scale fluid problems with small dissipation and the fluid regions are thin compared to the horizontal length scales. In this situation, one would like to know to what extent two dimensional models are appropriate and how one might incorporate three dimensional effects into such a model (for shallow-water problems, see [CaHo], [Ol], for example).

Such problems lead to interesting mathematical questions. While global existence and uniqueness of classical solutions of the Euler equations in two-dimensional domains is well known ([Li], [Wo], [Ka1], $[\mathrm{Yu}]$ ), only local existence (and uniqueness) of solutions is known in the threedimensional case ([EbMa], [Ka2], [Te]). When the three-dimensional domain is thin in one direction, one can hope to show that the time of existence of the solutions tends to infinity, when the thickness $\varepsilon$ of the domain goes to zero, and also, to compare these solutions to those of appropriate two-dimensional systems. In [MaRaRa1] and [MaRaRa2], we show that such expected properties are indeed true, by using standard estimates for the Euler equations combined with thin domains techniques as developed in [HaRa1], [HaRa2] and [RaSe1]. In particular, the mean value operator in the thin direction (see [HaRa1], [HaRa2]) plays an important role.

In this paper we present some of these results, emphasizing estimates between the solutions of 
the three-dimensional Euler equations and an asymptotic expansion in terms of two dimensional vector fields. As expected, the order zero term in this expansion is the solution of the limiting two-dimensional Euler equation. The proofs in [MaRaRa2] indicate that, in general, threedimensional effects beyond the two-dimensional Euler equations need to be included to obtain control on the error estimates for this asymptotic expansion. Taking into account the first order term in this expansion allows us, in contrast to [Ol], to choose initial data $U(0)$ for which the mean value of the derivative in the thin direction need not be small (Theorem 1 below). In this sense, our data are not nearly two-dimensional. We cannot prove that the solutions of the threedimensional Euler equations exist globally, when the thickness $\varepsilon>0$ is small but fixed. This feature is in contrast with the properties of the Navier-Stokes equations on three-dimensional thin domains, where existence of global solutions for large initial data is proved ([RaSe1], [RaSe2], [TeZi] and [IfRa]). In [MaRaRa2], we focus on two types of thin domains $Q_{\varepsilon}$ : cylindrical domains and spherical shells. The cylindrical case is easier because the two-dimensional Euler equations obviously embed into the three-dimensional ones. Because of limited space, we only describe the cylindrical case.

Consider the thin cylinder $Q_{\epsilon}=\Omega \times(0, \epsilon)$, where $\varepsilon$ is a small positive parameter, $\Omega=$ $\left[0, l_{1}\right] \times\left[0, l_{2}\right]$ and $l_{1}, l_{2}$ are two positive fixed numbers. We take $x_{1}$ and $x_{2}$ to be periodic variables with period $l_{1}$ and $l_{2}$ respectively and, correspondingly, we may regard $\Omega$ as a two torus. Thus, the boundary $\partial Q_{\varepsilon}$ of $Q_{\varepsilon}$ is given by $\Gamma_{0} \cup \Gamma_{\varepsilon}$ where $\Gamma_{0}=\Omega \times\{0\}$ and $\Gamma_{\varepsilon}=\Omega \times\{\varepsilon\}$. The vertical variable is denoted $x_{3}$. If the velocity field of the fluid on the thin domain $Q_{\varepsilon}$ and the pressure are denoted by $U=\left(U_{1}, U_{2}, U_{3}\right) \equiv\left(U^{*}, U_{3}\right)$ and $P$, respectively, the Euler equations are given by

$$
\begin{gathered}
\frac{\partial U}{\partial t}+(U \cdot \nabla) U+\nabla P=0, \\
\operatorname{div} U=0, \\
U_{3}=0 \text { on } \Gamma_{0} \cup \Gamma_{\varepsilon}, \\
U \text { is periodic in } x_{1}, x_{2} \text { with periods } l_{1}, l_{2}, \\
U(\cdot, 0)=U^{0},
\end{gathered}
$$

where the initial condition $U^{0}$ belongs to the space

$$
\begin{array}{r}
X^{m, q}\left(Q_{\varepsilon}\right) \equiv\left\{U \in\left[W^{m, q}\left(Q_{\varepsilon}\right)\right]^{3} \mid \operatorname{div} U=0, U_{3}=0 \text { on } \Gamma_{0} \cup \Gamma_{\varepsilon},\right. \\
\left.U \text { is periodic in } x_{1}, x_{2} \text { with periods } l_{1}, l_{2},\right\}
\end{array}
$$

and where, for $m \in \mathbb{N}, q \geq 1, W^{m, q}\left(Q_{\varepsilon}\right)$ denotes the Sobolev space on $Q_{\varepsilon}$, equipped with the standard norm. Local existence theory shows that, if $q>1$ and $m>1+3 / q$, there is a $T_{*}>0$ and a unique solution $(U, P) \in \mathcal{C}^{0}\left(\left[0, T_{*}\right) ; X^{m, q}\left(Q_{\varepsilon}\right) \times W^{m+1, q}\left(Q_{\varepsilon}\right) / \mathbb{R}\right)$ of the Euler equations (1). In the sequel, we shall assume that $U^{0} \in X^{2, q}\left(Q_{\varepsilon}\right)$ for $q>3$.

In the next section, we describe the existence and comparison results for the solutions of Equation (1) in the cylindrical case.

\section{The thin cylindrical case}

The mean value operator in the vertical direction is defined as follows. For any function $f \in$ $L^{p}\left(Q_{\varepsilon}\right)$, where $p \geq 1$, let

$$
(M f)\left(x_{1}, x_{2}\right)=\frac{1}{\varepsilon} \int_{0}^{\varepsilon} f\left(x_{1}, x_{2}, s\right) d s .
$$


The mean value operator on vector fields is the bounded linear operator from $\left(L^{p}\left(Q_{\varepsilon}\right)\right)^{3}$ to $\left(L^{p}(\Omega)\right)^{3}$ defined by $\bar{M} U=\bar{M}\left(U_{1}, U_{2}, U_{3}\right)=\left(M U_{1}, M U_{2}, 0\right)$. Since the third component of $\bar{M} U$ is zero, we can identify $\bar{M} U$ with a 2-dimensional vector field.

To find the limiting equations on $\Omega$ in a systematic way, we formally expand $\left(U^{*}, U_{3}, P\right)$ in a power series relative to the variable $x_{3}$, that is,

$$
\begin{aligned}
U^{*} & =U_{0}^{*}+U_{1}^{*} x_{3}+\frac{1}{2} U_{2}^{*} x_{3}^{2}+\cdots, \\
U_{3} & =\frac{1}{2} U_{3,2} x_{3}^{2}+\cdots, \\
P_{0} & =P_{0}+P_{1} x_{3}+\frac{1}{2} P_{2} x_{3}^{2}+\cdots .
\end{aligned}
$$

Due to the boundary conditions on $U_{3}$, the first two terms $U_{3,0}$ and $U_{3,1}$ vanish. Inserting (2) into (1) and equating to zero every coefficient of $x_{3}^{k}, k=0,1$, yields the following equations, where $U_{i}^{*}, U_{3, i}$ and $P_{i}$ are periodic in the variables $x_{1}, x_{2}$,

- Order 0

$$
\frac{\partial U_{0}^{*}}{\partial t}+U_{0}^{*} \cdot \bar{\nabla} U_{0}^{*}=-\overline{\operatorname{grad}} P_{0}, \quad \overline{\operatorname{div}} U_{0}^{*}=0,
$$

as well as the equation $P_{1}=0$.

- Order 1

$$
\frac{\partial U_{1}^{*}}{\partial t}+\left(U_{1}^{*} \cdot \bar{\nabla}\right) U_{0}^{*}+\left(U_{0}^{*} \cdot \bar{\nabla}\right) U_{1}^{*}=0
$$

as well as the equalities $P_{2}=0$ and $U_{3,2}=-\overline{\operatorname{div}} U_{1}^{*}$.

Here, $\bar{\nabla}$ denotes the 2-dimensional gradient and $\overline{\text { div }}$ denotes the 2-dimensional divergence operator. The equations (3) are the 2-dimensional Euler equations on $\Omega$. As already noted, one has global existence of solutions and estimates ([Ka1], $[\mathrm{KaPo}]$ ). The system (4) for the 2 -dimensional vector field $U_{1}^{*}$ on $\Omega$ is linear and thus admits always global solutions. The vector field $U_{1}^{*}$ is the analogue of the director field in elasticity.

At order $k$, the equation for $U_{k}^{*}$ has the form $\frac{\partial U_{k}^{*}}{\partial t}+\left(U_{k}^{*} \cdot \bar{\nabla}\right) U_{0}^{*}+\left(U_{0}^{*} \cdot \bar{\nabla}\right) U_{k}^{*}=$ "known quantities", where the "known quantities" have been determined in the previous steps. Then, $U_{3, k+1}$ and $P_{k+1}$ are given algebraically in terms of the previously computed quantities.

Below, we denote by $\bar{U}_{0}^{*}$ and $\bar{U}_{1}^{*}$ the solutions of the equations (3) and (4) with initial data $\bar{U}_{0}^{*}=\bar{M} U^{0}$ and $\bar{U}_{1}^{*}=\bar{M}\left(\frac{\partial U^{0}}{\partial x_{3}}\right)$ respectively. Since $U^{0}$ belongs to $X^{2, q}\left(Q_{\varepsilon}\right)$, for $q>3$, $\left(\bar{U}_{0}^{*}(t), \bar{U}_{1}^{*}(t)\right)$ belongs to $\mathcal{C}^{0}\left([0,+\infty) ;\left(W^{2, q}(\Omega)\right)^{2} \times\left(W^{1, q}(\Omega)\right)^{2}\right)$.

We can now state the main theorem for thin cylindrical domains, which is proved in [MaRaRa2]. The hypotheses will be as follows:

H1 Let $q>3, K_{0}>0$ be given. Suppose that $\gamma$ and $\alpha$ are positive numbers satisfying:

$$
0 \leq \gamma<\alpha<\min \left(\frac{q-3}{q}, \frac{(2 q+5)(q-3)^{2}+14(q-3)+1}{q^{2}(3 q-4)}\right) .
$$

H2 The following conditions on the initial data hold: 


$$
\begin{aligned}
& \max \left(\left\|\bar{M} U^{0}\right\|_{W^{2, q}(\Omega)},\left\|\bar{M} \frac{\partial U^{0}}{\partial x_{3}}\right\|_{L^{\infty}(\Omega)}\right) \leq K_{0}, \\
& \max \left(\left\|U^{0}\right\|_{W^{2, q}\left(Q_{\varepsilon}\right)},\left\|\bar{M} \frac{\partial U^{0}}{\partial x_{3}}\right\|_{W^{1, q}(\Omega)}\right) \leq K_{0} \varepsilon^{-\gamma} .
\end{aligned}
$$

Theorem 1. Assume that the hypotheses $\mathbf{H 1}$, H2 hold. Then there are positive numbers $\varepsilon_{0}=$ $\varepsilon_{0}\left(q, K_{0}, \gamma, \alpha\right)$ and $\delta=\delta\left(q, K_{0}, \gamma, \alpha\right)$ and positive constants $C_{1}=C_{1}\left(q, K_{0}, \alpha, \gamma\right)$ and $\tilde{C}=\tilde{C}\left(K_{0}\right)$ such that, for $\varepsilon \in\left[0, \varepsilon_{0}\right]$, the Euler equations (1) on $Q_{\varepsilon}$ have a unique solution $(U(t), P(t)) \in$ $\mathcal{C}^{0}\left([0, \tau(\varepsilon)] ; X^{2, q}\left(Q_{\varepsilon}\right) \times W^{3, q}\left(Q_{\varepsilon}\right) / \mathbb{R}\right)$, where $\tau(\varepsilon)$ satisfies

$$
\tau(\varepsilon) \geq \tilde{C} \log (\log [-\log \varepsilon]) .
$$

Moreover, the following estimates hold for $0 \leq t \leq \tau(\varepsilon)$ :

$$
\|U(t)\|_{W^{2, q}\left(Q_{\varepsilon}\right)} \leq 3 K_{0} \varepsilon^{-\alpha}
$$

and

$$
\left\|\bar{\nabla}\left(U-\bar{U}_{0}^{*}\right)(t)\right\|_{L^{\infty}\left(Q_{\varepsilon}\right)}+\left\|\left(\frac{\partial U^{*}}{\partial x_{3}}-\bar{U}_{1}^{*}\right)(t)\right\|_{L^{\infty}\left(Q_{\varepsilon}\right)}+\left\|\frac{\partial U_{3}}{\partial x_{3}}(t)\right\|_{L^{\infty}\left(Q_{\varepsilon}\right)} \leq C_{1} \varepsilon^{\delta} .
$$

The proof of Theorem 1 relies on a contradiction argument, on a priori estimates involving the quantity $\|\nabla U(t)\|_{L^{\infty}\left(Q_{\varepsilon}\right)}$ similar to those of [BeKaMa], [Fe], [KaPo] and on the comparison of the vector fields $\bar{M} U(t)$ with $\bar{U}_{0}^{*}(t)$ and $\bar{M}\left(\frac{\partial U}{\partial x_{3}}\right)(t)$ with $\bar{U}_{1}^{*}(t)$ in the $L^{\infty}$-norm on $\Omega$. To bound the terms $(\operatorname{Id}-\bar{M}) U(t)$ and $(\operatorname{Id}-\bar{M})\left(\frac{\partial U}{\partial x_{3}}\right)(t)$ in the $L^{\infty}$-norm on $Q_{\varepsilon}$, we use Poincaré inequalities and exploit the divergence free condition of $U(t)$.

Remarks 1. Theorem 1 is still true if the constant $K_{0}$ in the hypothesis $\mathbf{H 2}$ is replaced by $K_{0} f(\varepsilon)$, where $f(\varepsilon)$ is a positive function of $\varepsilon$, which goes very slowly to infinity, as $\varepsilon$ goes to zero. For instance, we can choose $f(\varepsilon)=\log (\log (\log [-\log \varepsilon]))$. The hypothesis $\mathbf{H 1}$ can also be made weaker (see $[\mathrm{MaRaRa}]$ ).

2. If the initial data $U^{0}$ are bounded in $\left(W^{3, p}\left(Q_{\varepsilon}\right)\right)^{3}, p \geq 2$ for example, one shows that $\|U(t)\|_{W^{3, p}\left(Q_{\varepsilon}\right)}$ satisfies the estimate (5) on the time interval $[0, \tau(\varepsilon)]$, by using Theorem 1 and taking into account the terms of order 2 in the expansion (2). Of course, one obtains estimates, similar to (6), involving $\bar{U}_{0}^{*}(t), \bar{U}_{1}^{*}(t)$ and $\bar{U}_{2}^{*}(t)$, where $\bar{U}_{2}^{*}(t)$ is the vector field appearing in (2) at order two and satisfying $\bar{U}_{2}^{*}(0)=\bar{M}\left(\frac{\partial^{2} U^{0}}{\partial x_{3}^{2}}\right)$ (see [MaRaRa2]). The estimate of the derivatives of order $k \geq 3$ of $U(t)$ requires the study of the terms in the expansion (2) up to order $k-1$, which becomes rather technical, when $k$ increases.

3. In [MaRaRa1], we have described existence and comparison results in the spherical case, when $U^{0}$ is in $\left(H^{3}\left(Q_{\varepsilon}\right)\right)^{3}$. To prove the existence of the solution $U(t)$ on $[0, \tau(\varepsilon)]$, we took into account the expansion (2) up to order two. [MaRaRa2] simplifies this part of the proof since now one only needs to work with the first two vector fields $\bar{U}_{0}^{*}$ and $\bar{U}_{1}^{*}$.

4. Similar results to those of Theorem 1 hold if $Q_{\varepsilon}=\left\{\left(x_{1}, x_{2}, x_{3}\right) \mid\left(x_{1}, x_{2}\right) \in \Omega, 0<x_{3}<\right.$ $\left.\varepsilon g\left(x_{1}, x_{2}\right)\right\}$, where $g\left(x_{1}, x_{2}\right)$ is a positive function, periodic in $x_{1}$ and $x_{2}$ of period $l_{1}$ and $l_{2}$ respectively. This case is studied in [Ol], who proved existence of a solution $U(t)$ on long time intervals and compared $U(t)$ with a solution of the 2-dimensional Euler equations, assuming that the data $U^{0}$ are nearly 2-dimensional in $X^{m, 2}\left(Q_{\varepsilon}\right)$ for $m \geq 3$. In the above situation, he 
had to assume that, for $0 \leq j \leq 2,\left\|\bar{M} D^{j}\left(\frac{\partial U^{0}}{\partial x_{3}}\right)\right\|_{H^{m}\left(Q_{\varepsilon}\right)} \leq \delta \varepsilon^{1 / 2}$, where $\delta$ is sufficiently small and showed that $\left\|U(t)-\bar{U}_{0}^{*}(t)\right\|_{H^{m}\left(Q_{\varepsilon}\right)} \leq C \varepsilon^{1 / 2}$ on some time interval. Since, in our case, $\delta$ can be large, we need to take into account the vector field $\bar{U}_{1}^{*}$.

5. In [MaRaRa2], Theorem 1 is generalized to the case of a thin spherical shell, that is, the domain bounded by two spheres of radii $r=1$ and $r=1+\varepsilon$. Again, we have to consider the asymptotic expansion (2), where the variable $x_{3}$ becomes $\rho=r-1$. Thus, $U_{0}^{*}$ is a solution of the Euler equations on the sphere $S^{2}$, while $U_{1}^{*}$ is the solution of a "linearized" Euler-type equation on $S^{2}$. The geometry of $Q_{\varepsilon}$ appears through the fact that now $P_{0}=k\left(U_{0}^{*}, U_{0}^{*}\right)$ and $P_{1}=k\left(U_{0}^{*}, U_{0}^{*}\right)+2 k\left(U_{0}^{*}, U_{1}^{*}\right)$, where $k(\cdot, \cdot)$ is the second fundamental form on $S^{2}$.

6 . In the context of rotating fluids, Babin, Mahalov and Nicolaenko [BaMaNi] showed existence of solutions of the Euler equations with an additional Coriolis term, on a time interval $[0, T(R)]$, where $T(R)$ goes to infinity as the Rossby number $R$ goes to zero.

\section{References}

[BaMaNi] A. Babin, A. Mahalov and B. Nicolaenko, Regularity and integrability of 3D Euler and Navier-Stokes equations for rotating fluids. Asymptotic Anal. 15, (1997), 103-150.

[BeKaMa] J.T. Beale, T. Kato, and A. Majda, Remarks on the breakdown of smooth solutions for the 3D Euler equations. Comm. Math. Phys. 94, (1984), 61-66.

[CaHo] R. Camassa and D.D. Holm, Dispersive barotropic equations for stratified mesoscale ocean dynamics. Physica D 60, (1992), 1-15.

[EbMa] D.G. Ebin and J.E. Marsden, Groups of diffeomorphisms and the motion of an incompressible fluid, Ann. Math. 92, (1970), 102-163.

[Fe] A.B. Ferrari, On the blow-up of solutions of the 3-D Euler equations in a bounded domain Comm. Math. Phys. 155, (1993), 277-294.

[HaRa1] J.K. Hale and G. Raugel, Partial differential equations on thin domains, Differential Equations and Mathematical Physics, (Birmingham, AL, 1990), C. Bennewitz ed., Academic Press, (1992), 63-98.

[HaRa2] J.K. Hale and G. Raugel, Reaction-diffusion equations on thin domains, Journ. Math. Pures Appl. 71, (1992), 33-95.

[IfRa] D. Iftimie and G. Raugel, Some results on the Navier-Stokes equations in thin 3D domains, J. Differential Equations, to appear.

[Ka1] T. Kato, On classical solutions of the two-dimensional non-stationary Euler equation, Arch. Rat. Mech. Anal. 25, (1967), 188-200.

[Ka2] T. Kato, Nonstationary flows of viscous and ideal fluids in $\mathbb{R}^{3}$, J. Funct. Anal. 9, (1972), 296-305.

[KaPo] T. Kato and G. Ponce, Well-posedness of the Euler and Navier-Stokes equations in the Lebesgue spaces $L_{s}^{p}\left(\mathcal{R}^{2}\right)$, Rev. Mat. Iberoam. 2, (1987), 73-88.

[Li] L. Lichtenstein, Über einige Existenzprobleme der Hydrodynamik homogener unzusammendrückbarer, reibungsloser Flüssigkeiten und die Helmholtzschen Wirbelsätze, Math. Z. 23, (1925), 89-154, 309-316, 26, (1927), 196-323, 28, (1928), 387-415, 725, 32, (1930), 608.

[MaRaRa1] J. E. Marsden, T. Ratiu and G. Raugel, Equations d'Euler dans une coque sphérique mince, C.R. Acad. Sci. Paris 321, (1995), 1201-1206. 
[MaRaRa2] J. E. Marsden, T. Ratiu and G. Raugel, The Euler equations on three-dimensional thin domains, manuscript, (1999).

[Ol] M. Oliver, Justification of the shallow-water limit for a rigid-lid flow with bottom topography, Theoretical and Comput. Fluid Dynamics 9, (1997), 311-324.

[RaSe1] G. Raugel and G.R. Sell, Navier-Stokes equations in thin 3D domains I: Global attractors and global regularity of solutions, Journ. Amer. Math. Soc., 6, (1993), 503-568.

[RaSe2] G. Raugel and G.R. Sell, Navier-Stokes equations on thin 3D domains. II. Global regularity of spatially periodic solutions. Nonlinear partial differential equations and their applications, Collège de France Seminar, Vol. XI , (1994), 205-247, Pitman Res. Notes Math. Ser., 299, Longman Sci. Tech., Harlow.

[Te] R. Temam, On the Euler equations of incompressible perfect fluids J. Funct. An. 20, (1975), $32-43$.

[TeZi] R. Temam and M. Ziane, Navier-Stokes equations in three-dimensional thin domains with various boundary conditions, Adv. Differential Equations 1, (1996), 499-546.

[Wo] W. Wolibner, Un théorème sur l'existence du mouvement plan d'un fluide parfait, homogène, incompressible, pendant un temps infiniment long, Math. Z. 37, (1933), 698-726.

[Yu] V.I. Yudovich, Nonstationary flows of an ideal incompressible fluid. Zh. Vych. Mat. 6, (1973), 1032-1066. 